\title{
¿Cómo clasificar las actividades policiales y evaluar la orientación práctica de la policía?
}

\section{How to classify police activities and evaluate the practical orientation of the police Como classificar as atividades policiais e avaliar a orientação prática da polícia?}

\author{
Augusto Rigoberto López Ramírez * \\ https://orcid.org/0000-0002-5769-1717 Universidad Centroamericana José Simeón Cañas, \\ Antiguo Cuscatlán, El Salvador
}

- Fecha de recepción: 2020-11-19

- Fecha concepto de evaluación: 2021-01-28

- Fecha de aprobación: 2021-02-10 https://doi.org/10.22335/rlct.v13i2.1340
Para citar este artículo / To reference this article / Para citar este artigo: López, A. R. (2021). ¿Cómo clasificar las actividades policiales y evaluar la orientación práctica de la policía? Revista Logos Ciencia \& Tecnología, 13(2), 24-38. https://doi.org/10.22335/rlct.v13i2.1340

\section{RESUMEN}

Se presenta una propuesta general para clasificar actividades desarrolladas por patrullas territoriales de policía. El artículo tiene por objetivo exponer la clasificación de las actividades para proponerla a investigadores, jefes de policía y tomadores de decisión como una alternativa práctica y útil para realizar análisis de operatividad policial. Utilizando esta clasificación se han evaluado los siguientes aspectos: a) el posicionamiento de la policía frente a la realidad y su objeto de acción; b) el trato de la policía con la ciudadanía y c) la implementación de un programa de policía comunitaria en El Salvador. La propuesta se ha elaborado a partir de dos investigaciones realizadas en El Salvador hechas con metodología mixta. Los resultados muestran la puesta en práctica de la clasificación y las posibilidades que esta ofrece para facilitar evaluaciones de cuerpos policiales en los aspectos señalados. Puede concluirse que esta clasificación cumple su cometido analítico y habilita nuevas posibilidades para profundizar en estudios del desempeño policial. El artículo finaliza proporcionando pistas analíticas para la interpretación de los datos a fin de motivar futuras investigaciones.

Palabras clave: administración policial, evaluación de la policía, funciones de la policía, organización policial, selectividad de la acción policial 


\begin{abstract}
A general proposal is presented to classify police activities developed by territorial patrols. This paper aims to present the classification of activities and propose it to investigators, police chiefs and decision makers as a practical and useful alternative to carry out analysis of police operations. Using this classification, the following aspects have been evaluated: a) the position of the police in the face of reality and its object of action; b) the treatment of the police with citizens; and c) the implementation of a community policing program in El Salvador. The proposal has been elaborated from two researches carried out in El Salvador with mixed methodology. The results show the implementation of the classification and the possibilities it offers to facilitate evaluations of police forces in the indicated aspects. It can be concluded that this classification fulfills its analytical role and opens up new possibilities for deepening studies of police performance. The article ends by providing analytical clues for the interpretation of data in order to motivate future research.
\end{abstract}

Keywords: police administration, police evaluation, police functions, police organization, selectivity of police action

\title{
RESUMO
}

É apresentada uma proposta geral para classificar as atividades realizadas pelas patrulhas policiais territoriais. O artigo tem como objetivo apresentar a classificação das atividades para propô-la a investigadores, chefes de polícia e tomadores de decisão como uma alternativa prática e útil para a realização de análises de operações policiais. Com base nessa classificação, foram avaliados os seguintes aspectos: a) a posição da polícia em relação à realidade e seu objeto de ação; b) o tratamento da polícia com os cidadãos; e c) a implementação de um programa de polícia comunitária em El Salvador. A proposta foi elaborada a partir de duas pesquisas realizadas em El Salvador com metodologia mista. Os resultados mostram a implementação da classificação e as possibilidades que ela oferece para facilitar a avaliação das forças policiais nos aspectos indicados. Pode-se concluir que essa classificação cumpre o seu papel analítico e abre novas possibilidades para o aprofundamento nos estudos da atuação policial. O artigo finaliza fornecendo dicas de análise para a interpretação dos dados a fim de motivar pesquisas futuras.

Palavras-chave: administração policial, avaliação da polícia, funções policiais, organização policial, seletividade da ação policial

Este artículo sistematiza la experiencia analítica y metodológica de dos investigaciones realizadas en El Salvador en el campo de los estudios de policía. Tales experiencias presentan una característica común: en ellas se recolectaron datos de las actividades policiales operativas a fin de examinar su orientación.

Se construyeron cuatro categorías originales de actividades: a) actividades preventivas-proactivas; b) actividades preventivas-reactivas; c) actividades represivas-proactivas; y d) actividades represivas-reactivas. Esta clasificación permitió evaluar los siguientes aspectos de la actividad policial: a) el posicionamiento de la policía frente a la realidad y su objeto de acción; b) el trato de la policía con la ciudadanía; y c) la implementación de un programa de policía comunitaria en El Salvador.
La sistematización de estas experiencias se hace con el objetivo de proponer a investigadores, jefes de policía y tomadores de decisión una alternativa práctica y útil para realizar el análisis de la operatividad policial, aspecto sobre el cual reside buena parte de la efectividad institucional (Famega, 2003).

La categorización de actividades propuesta no pretende ser exhaustiva; sin embargo, ofrece una alternativa de análisis bien fundamentada que permite discutir las prácticas policiales en cuanto a su orientación en aspectos relevantes para la sociedad y la policía. En esta introducción se expondrá un estado del arte sobre cómo se clasifican las actividades policiales y cómo se ha llegado a la propuesta original de este artículo. 
En realidad, hay muchas investigaciones que se dedican a examinar las actividades de la policía y, sobre todo, predominan los estudios situados en Estados Unidos, aunque también hay esfuerzos sistemáticos desde América Latina que se ubican en el campo más amplio de la evaluación policial (Cano, 2003; Mohor, 2007).

Generalmente, suele clasificarse a las actividades policiales en razón de las funciones organizativas que le son delegadas a la policía. Al respecto, prevalece una división entre funciones preventivas y funciones de control (Famega, 2003), o en otras palabras, entre funciones de mantenimiento del orden y funciones para hacer cumplir la ley.

Esa clasificación es un lugar común en los estudios de policía (Cano, 2003; Famega, 2003; Webb \& Katz, 1997; Wilson, 1968) que se asume como una dualidad inherente del trabajo policial, pues se deriva de su característica como fuerza pública. En este sentido, la policía es tanto un instrumento para distribuir fuerza como un instrumento para prevenir delitos y brindar servicios, en la medida que su disponibilidad lo permite (Monjardet, 2010).

También es un denominador común afirmar que la característica principal de la policía, frente a otras instancias de servicio y control, es su autorización para hacer uso de la fuerza en el territorio nacional - fundamentalmente en el espacio público-, con superioridad frente a otros actores armados y de manera generalizada en la ciudadanía y no focalizada en grupos específicos (Bayley, 2010; Monjardet, 2010).

Sin embargo, tal prerrogativa no está pensada para subyugar a la población, sino para garantizar los derechos y libertades de esta. Es así que la policía tiene tres fuentes de acción: a) las solicitudes de la ciudadanía, b) las órdenes jerárquicas derivadas por vía organizativa, y c) la iniciativa profesional de los agentes de policía (Monjardet, 2010). Y, además, desarrolla tres procesos básicos (Aparicio, 2017): prevención, investigación e inteligencia.

La clasificación dual, mencionada párrafos atrás, ha predominado en buena parte de las investigaciones sobre instituciones policiales en los países desarrollados y también en América Latina, lo cual empata con el esfuerzo académico creciente para evaluar a los cuerpos policiales, es decir, verificar según marcos analíticos particulares si el accionar policial se apega o no a lo esperado, tanto desde un interés académico como desde el ámbito de la administración pública.
Debe señalarse que no hay consenso acerca de lo que significa la eficacia policial (Sung, 2006). Se sabe que la policía es un instrumento de control y un instrumento de servicio, pero no la medida del éxito de tales dimensiones, fundamentalmente porque la policía no es neutral en su accionar (Reiner, 2012) y ello plantea ambigüedades, dependiendo de la perspectiva del actor interesado.

La policía no es neutral, porque ayuda y reprime en una misma circunstancia; eso plantea la necesidad de definir múltiples indicadores de su éxito y no solo uno. Adicionalmente, el objeto de acción de la policía, la conflictividad social violenta, no es una dimensión de la realidad que responda exclusivamente a las acciones policiales, pues tiene su propia dinámica y se configura solo en parte por lo que la policía haga o deje de hacer (Bayley, 2010; Dadds \& Scheide, 2000; Travis, 1997).

Lo anterior nos regresa al debate acerca de la naturaleza de la función policial y de cómo debería evaluarse: ¿cómo un proceso o cómo un producto? Sung (2006) sostiene que la función policial no está expresada en términos absolutos sino en análisis relativos. Se sabe que la policía no podrá controlar la delincuencia ni suprimir el desorden violento por completo, pero sí deberían esperarse de ella ciertos grados de eficacia. Es decir, el análisis de eficacia debería basarse, más bien, en una discusión de grados y avances (Sung, 2006).

Así, la policía estaría inmersa en un proceso de producción de seguridad (Sung, 2006) planteado en términos más dinámicos. De esta forma, suelen identificarse algunas áreas de medición sobre las cuales la policía podría tener cierto dominio. Pese a las variaciones entre autores, estas áreas son comunes en sus aspectos medulares (Travis, 1997):

- Dominio de impacto: indicadores delictivos, miedo al delito y desorden (un ámbito donde la policía tiene limitada incidencia).

- Dominio de salud organizacional: satisfacción interna del trabajo policial, apoyo de la comunidad, conocimiento del entorno de trabajo.

- Dominio de los procesos: proporción de un servicio equitativo, proporción de un servicio íntegro, o desviado, habilidades para el comportamiento ético, legitimidad desde la perspectiva ciudadana (este dominio da cuenta de cómo la policía hace su trabajo).

- Dominio del contexto comunitario: cohesión social de los territorios, control social informal, estructuras polí- 
ticas y económicas (un ámbito donde la policía tiene limitada incidencia).

Las evaluaciones centradas en estos dominios continúan recogiendo en el fondo las dos dimensiones de la dualidad policial: el control (indicadores delictivos) y el servicio (satisfacción de la ciudadanía).

Una perspectiva alternativa es la que se enfoca en las actividades que realiza la policía, es decir, la dimensión operativa sobre lo que hace. Aquí pueden recogerse datos sobre detenciones, decomisos de ilícitos, Ilamadas de emergencias atendidas, tiempo de respuesta a una emergencia, enfrentamientos armados, aplicaciones de la fuerza letal, entre otras actividades de interés, dependiendo del contexto.

Esta variante de la evaluación trata de concentrarse en lo que realmente controla la policía: sus acciones, cuyo examen puede dar paso a monitorear, evaluar y tomar decisiones en general. En otro tipo de mediciones esto es limitado, porque se enfocan en resultados que no dependen directamente de la acción policial, por ejemplo, los índices delictivos.

También pretende aproximarse a los mecanismos de la actuación policial, no tanto en los insumos ni en los productos, sino en el cómo, es decir, en las prácticas que son el medio para alcanzar lo que se propone.

A juicio de Dadds \& Scheide (2000), Travis (1997) y Leggett (2003), la medición de actividades debería centrarse en datos fáciles de recolectar — que ya registran o podrían registrar las policías-, útiles para hacer inferencias sobre aspectos que la policía realmente controla y permitan análisis para que se propicien mejores prácticas dentro de la organización.

Así pueden identificarse distintas fuentes de recolección: encuestas a policías, boletas de autoregistro al finalizar turnos, órdenes al iniciar turnos, observación de actividades in situ y encuestas a la ciudadanía acerca de las prácticas policiales. También, existen diferentes énfasis sobre los focos de atención para analizar las acciones: la frecuencia, el tiempo que requieren, la fuente de acción, la carga laboral del personal, entre otras.

Hay investigaciones que se dirigen a comparar, por medio de las actividades policiales, si algunos programas de policía se implementan en la práctica y cómo lo hacen, por ejemplo, al examinar las actividades que realizan pa- trullas ordinarias y patrullas dedicadas a la prevención comunitaria (Webb \& Katz, 1997). O bien, investigaciones que miden los procesos policiales básicos: actividades preventivas, de investigación o de inteligencia (Monjardet, 2010).

Es muy común que las actividades policiales se midan en razón del tiempo que ocupan en los turnos de trabajo de los policías; a esos estudios se les denomina de carga laboral y se basan en monitorear el tiempo en relación a una variedad de actividades o productos, estableciendo un vínculo entre la cantidad de tiempo y los resultados (Dadds \& Scheide, 2000).

Lo anterior permite examinar la asignación de recursos, evaluar los costos que implica tener ciertos resultados, contribuye a fijar objetivos y provee información útil a la ciudadanía sobre lo que realmente hace la policía (Dadds \& Scheide, 2000).

Sin embargo, un problema presente en todos los estudios es la clasificación de las actividades y su agrupamiento por homogeneidad de acuerdo con algunos criterios. En ese ejercicio los grupos de actividades suelen ser muy diversos y hasta ambiguos, se mezclan las actividades entre ellos y es evidente la ausencia de criterios estables (Dadds \& Scheide, 2000; Leggett, 2003; Webb \& Katz, 1997).

Famega $(2003 ; 2005)$ sostiene que en la mayoría de investigaciones yace la clasificación tradicional entre actividades de control (relacionadas a delitos) y actividades de servicio y prevención, obviando dos dimensiones de análisis poco exploradas en los estudios de policía, pero que están presentes en el día a día de su labor: las acciones reactivas y las acciones proactivas.

Más allá de medir las actividades en razón del tiempo que ocupan en la jornada laboral, Famega (2003) propone que el análisis debería centrarse en su dirección, a fin de superar lugares comunes en los hallazgos, tales como: los policías tienen mucho tiempo no comprometido, se dedican más a actividades de servicio y administrativas, y las actividades de control delictivo representan una pequeña porción de su tiempo (Frank et al., 1997). Pero, para superarlos, deberían crearse focos de análisis distintos. En este artículo se retoma la preocupación de Famega (2003) por hacer visible las dimensiones proactivas y reactivas de la acción policial, a la vez que las dimensiones de servicio y de control. 
Adicionalmente, se agregará una acepción nueva a los conceptos de actividades de servicio y de control, las cuales, como se ha visto, se refieren tradicionalmente a acciones con o sin implicación penal.

Generalmente, se consideran actividades de servicio aqueIlas que se aplican sobre situaciones que plantean conflictos de orden público (convivencia, disputas civiles, molestias públicas) (Sung, 2006) o emergencias varias, donde la policía interviene de manera autorizada en tanto fuerza superior y como organización auxiliar con capacidad de respuesta; por su parte, las acciones de control se realizan en el campo de las situaciones que tienen implicaciones penales, ya sea de procesos activos o donde se presume puede haber consecuencias de este tipo.

Como se verá más adelante, la frontera entre estos dos grupos es difusa, porque una acción de servicio puede terminar en una implicación penal o una acción de control, donde se presumen consecuencias penales, puede resolverse sin necesidad de conducirla a ese escenario. Al respecto, debe considerarse el antes y después de la acción: cuando se ordena o solicita, y cuando se informa de su realización, siendo más confiables los datos a posteriori.

Pero esa clasificación dicotómica deja por fuera el trato con la ciudadanía, el cual está mediado por la posibilidad de que la policía pueda hacer uso de la fuerza. Frente a ello, en lugar de utilizar los conceptos de servicio y control, aquí se prefiere utilizar los conceptos de acciones preventivas y acciones represivas.

Vale la pena hacer una diferencia de acepciones en el lenguaje, porque en los estudios policiales publicados en inglés es más usual que se utilicen términos como: law enforcement, para las acciones penales, y call service, peace keeping u order manteinence, para las acciones de servicio. En español, principalmente en América Latina y, de manera especial en El Salvador, los términos de prevención y represión son más familiares.

Pero, además, estos últimos conceptos incorporan un elemento más patente de uso de fuerza, porque la represión conlleva la coacción (fuerza, violencia) de comportamientos y la prevención implica un momento no coactivo, en el que las relaciones no están predispuestas para tal uso efectivo de la fuerza.

En tal sentido, los conceptos que se utilizan para clasificar las actividades en este documento son:
1. Acciones preventivas: son aquellas que no tienen implicación penal o no tienen predisposición al uso de la fuerza, es decir, son no coactivas.

2. Acciones represivas: son aquellas que tienen una implicación penal o tienen predisposición al uso de la fuerza, es decir, son coactivas en su interacción.

Asimismo, se usan los conceptos de acciones proactivas -aquellas que se originan por iniciativa del policía operativo o la organización policial- y acciones reactivas aquellas que se originan en el entorno, o a solicitud de la ciudadanía, y la policía solo se dispone para atenderlas-.

Sin embargo, hay características de la acción policial que la tornan compleja para ser capturada por conceptos que pretendan medirla. En realidad, las acciones policiales son un continuo y su discriminación suele ser arbitraria (Carrington \& Schulenberg, 2003), de modo que, incluso precisando los conceptos anteriores, existen al menos las siguientes dificultades identificadas:

a. Actividades marco donde caben muchas otras, tales como el patrullaje a pie y el patrullaje en vehículo.

b. Actividades que inician de una forma, pero pueden devenir en otra, tales como atender una solicitud por problemas de convivencia entre vecinos que termina en agresiones físicas con consecuencias penales.

c. Actividades de acción corta, como recibir una denuncia o realizar un registro, y actividades de acción larga, como una reunión comunitaria o una charla en un centro educativo, y que por tanto registran menos frecuencia.

d. Actividades que son difíciles de precisar por su ambigüedad, como por ejemplo, el propiciar conversaciones con la ciudadanía, que puede comprender un acercamiento cotidiano para ganar familiaridad o pretender indagar indirectamente sobre hechos delictivos.

e. Actividades que se solapan entre sí, como las visitas casa a casa que pueden ser acompañadas (en El Salvador) de operativos de cierre para controlar la movilidad humana circundante.

Todo lo anterior invita a hacer algunas acotaciones, que se tuvieron presentes para realizar la clasificación que se presenta en este artículo:

a. Se tomaron en cuenta solo actividades específicas y no actividades marco.

b. Se tomó en consideración la valoración de la actividad después de su realización. 
c. No se consideró la duración temporal de las actividades, sino su frecuencia delimitada a un periodo: la intensidad de la actividad en una semana, para efectos comparativos.

d. Se seleccionaron aquellas actividades más representativas del tipo de aspectos que se intentaba evaluar, pues no se trata de una evaluación de la carga laboral total, teniendo en cuenta que hay actividades muy frecuentes, pero que no aportan a la evaluación y podrían contaminar el análisis, como por ejemplo, brindar seguridad en eventos o cuidar instalaciones.

e. Se complementó la información cuantitativa de las actividades con otra de tipo cualitativa, considerando también las opiniones de la ciudadanía. Por ejemplo, las actividades represivas también es necesario contrastarlas con las percepciones de justicia procedimental de la población (Tyler, 2004).

f. Solo se consideraron las acciones realizadas por la policía de seguridad (generalmente unidades de patrullas territoriales o de proximidad) (Monjardet, 2010), descartando a las unidades de investigaciones, de inteligencia y el personal dedicado a tareas administrativas.

Finalmente, con el propósito de recuperar las dos dimensiones de las actividades mencionadas (preventivorepresivo y reactivo-proactivo), y teniendo en cuenta las consideraciones para evitar la ambigüedad, en este artículo se propone una clasificación de cuatros grupos de actividades, de la siguiente manera:

a. Actividades preventivas-reactivas: se caracterizan por no estar relacionadas con asuntos penales ni tener predisposición al uso de la fuerza; además, su fuente de acción es externa y la policía solo se dispone a responder a ella por solicitud o de manera circunstancial. Son acciones de servicio requeridas por la ciudadanía e intervenciones en conflictos de convivencia.

b. Actividades preventivas-proactivas: se caracterizan por no estar relacionadas con asuntos penales ni tener predisposición al uso de la fuerza, pero en este caso la policía conserva la iniciativa y su fuente de acción es interna, tanto desde las órdenes jerárquicas como desde la iniciativa de policías operativos. Son acciones enmarcadas en programas de prevención social o de acercamiento a la comunidad.

c. Actividades represivas-reactivas: están relacionadas a situaciones con implicación penal y/o conllevan una predisposición al uso de la fuerza. Además, su fuente de acción es externa, y la policía solo responde a ellas por solicitud o de manera circunstancial. Son acciones frente a asuntos de naturaleza penal o intervenciones imperativas con la ciudadanía.

d. Actividades represivas-proactivas: están relacionadas a situaciones con implicación penal y/o conllevan una predisposición al uso de la fuerza. En este caso, la policía conserva la iniciativa y su fuente de acción es interna, tanto jerárquica como operativamente. Son acciones enmarcadas en programas de presencia policial intensiva, donde se realizan intervenciones imperativas.

En la tabla 1 se señalan algunos ejemplos de actividades que pueden incluirse en cada uno de los grupos recién señalados.

Tabla 1

Ejemplos de actividades que pueden incluirse en los grupos propuestos

Grupos de actividades Ejemplos

Atención de solicitudes relativas a problemas de convivencia (ya sea por solicitud a la línea telefónica o solicitud Actividades in situ), toma de denuncias sobre asuntos preventivas-reactivas de convivencia, atención de emergencias de salud de la ciudadanía, atención en accidentes de tránsito sin consecuencias penales.

\begin{tabular}{cl} 
& $\begin{array}{c}\text { Asistir o realizar reuniones con grupos } \\
\text { de ciudadanos; realizar visitas domicilia- } \\
\text { res; impartir charlas en centros escolares, } \\
\text { desarrollar programas de prevención } \\
\text { sctividades } \\
\text { social, atender factores de riesgo situa- } \\
\text { cionales de manera no coercitiva (ges- } \\
\text { tión de iluminación de calles, remoción } \\
\text { de vehículos abandonados, etc.), otras } \\
\text { acciones dirigidas a la resolución de } \\
\text { problemas comunitarios. }\end{array}$ \\
Actividades & $\begin{array}{l}\text { Capturas realizadas con orden judicial } \\
\text { yen flagrancia, atención de solicitudes } \\
\text { relativas a asuntos penales o donde } \\
\text { se antepone el uso de la fuerza física, } \\
\text { acciones de apoyo a investigaciones } \\
\text { penales, operativos de captura. }\end{array}$ \\
Actividades & $\begin{array}{l}\text { Registro (cacheo) e identificación } \\
\text { de personas, registro e identificación de } \\
\text { vehículos, fichaje (captura de datos per- } \\
\text { sonales), capturas derivadas de registros. }\end{array}$ \\
\hline
\end{tabular}

El propósito de este artículo es mostrar la puesta en práctica de esta clasificación, para evaluar tres aspectos de las acciones policiales: el primero se refiere al trato con la ciudadanía, que se obtiene de la dimensión represiva y preventiva; el segundo aspecto es el posicionamiento frente al objeto de acción que se obtiene de la dimensión proactiva y reactiva; el tercer aspecto se refiere a la im- 
plementación de un programa de policía comunitaria en El Salvador, que estaba en marcha al momento de realizar la recolección de datos.

En la siguiente sección, se detallará cómo se utilizó la clasificación anterior en dos investigaciones distintas, bajo qué condiciones se hizo y cómo se procesaron los datos para obtener la información deseada.

\section{Método}

El primer estudio en el cual se utilizó esta clasificación fue de carácter institucional, y fue elaborado por solicitud de la Academia Nacional de Seguridad Pública (ANSP) de El Salvador en el año 2019 (ANSP, 2019), buscando evaluar la actuación policial preventiva en el marco de la implementación de un programa de policía comunitaria que Ilevaba casi una década en funcionamiento. Vale aclarar que oficialmente se intentaba implementar una filosofía de policía comunitaria, pero en el estudio se identificaron actividades concretas para poder medirla: primero, para evaluar la actividad policial preventiva fue necesario delimitar qué se entendía por esto, haciendo diferencia fundamental entre lo preventivo-reactivo y lo preventivoproactivo. Lo preventivo-reactivo se entiende como la actividad policial tradicional en la que la policía se dispone a reaccionar, con el objetivo de disuadir o brindar servicios diversos; en general, los policías operativos consideran que el patrullaje en sí mismo es preventivo, cuando en realidad se trata de una idea de prevención limitada.

Se consideró que medir solamente esa dimensión implicaba presentar datos reducidos sobre la policía, limitando la prevención policial a la disuasión e incapacitación (Medina, 2013), extendiéndola en todo caso a la atención de emergencias ciudadanas e intervención en conflictos de convivencia.

De esta forma, se estimó necesario medir también las prácticas proactivas, las cuales encajan mejor con la idea que enarbolan ciertos programas policiales (como policía comunitaria y policía orientada a problemas y ventanas rotas) (Famega, 2003), en los cuales la policía conserva la iniciativa de la acción y puede desarrollar rutas para articular las situaciones de su interés (Manning, 2011), generalmente basadas en algún concepto preventivo del cual se tiene conciencia.
Adicionalmente, con el objetivo de lograr un ejercicio comparativo, también se indagó acerca de las actividades represivas-proactivas, que representan una categoría opuesta a la anterior, pues existe una predisposición al uso de la fuerza o bien se actúa sobre hechos delictivos consumados, todo ello mediado por una búsqueda consciente de la policía por realizar estas prácticas, persiguiendo objetivos de disuasión e incapacitación intensiva (Medina, 2013). Tales prácticas encajan con acciones que enarbolan ciertos programas policiales como: tolerancia cero, puntos calientes, Compstat (Famega, 2003).

Los datos cuantitativos fueron recolectados en una muestra de 470 policías del área de seguridad pública de tres delegaciones grandes (incluida la ciudad capital) por medio de una encuesta que se aplicó mediante autollenado en dos modalidades: en papel (44\%) y en formulario virtual (56\%) (ANSP, 2019). En todos los casos, las personas que participaron en la investigación lo hicieron de manera voluntaria y dando su consentimiento después de presentarles los objetivos y explicarles el resguardo y uso de los datos de la investigación.

Específicamente, se consultó a los entrevistados acerca de la intensidad con la que habían realizado ciertas actividades en la semana anterior a la aplicación de la encuesta, valorando esto en una escala de Lickert. Las opciones eran: nunca; casi nunca; algunas veces sí, algunas veces no; casi siempre; siempre. En una prueba de fiabilidad, esta escala obtuvo un alpha de Cronbach de 0.844; asimismo, en cuanto a validez de contenido mediante consulta a expertos, los ítems obtuvieron valores de 0.9 (V de Aiken) y 0.83 (Lawshe) (ANSP, 2019).

Por otra parte, el segundo estudio se trata de una investigación académica doctoral, que se basó en datos solicitados a la Policía Nacional Civil (PNC) por medio de la oficina de acceso a la información pública (PNC, 2020). Allí, se trabajó ajustándose al sistema de registro institucional de la policía, lo cual implicaba aceptar límites en cuanto a las actividades de las que se disponía, los datos y la manera en que la policía había conceptualizado las acciones.

En este estudio sobre prácticas policiales en dos colonias de San Salvador (colonia A y colonia B) se exploró la manera en la cual la policía se relacionaba con la ciudadanía y su posicionamiento frente a la realidad: la colonia $\mathrm{A}$ presentaba un escenario de control territorial consolidado de una pandilla y la colonia B era un caso de buenas prácticas en policía comunitaria. 
Se obtuvieron datos de las Ilamadas recibidas al sistema de emergencias telefónico 911, en las que la ciudadanía solicitaba acciones policiales, y sobre las cuales se dispuso personal para su atención, así como datos sobre operatividad de la patrulla policial asignada de manera permanente en los territorios y la información reportada diariamente por el personal policial.

Estos datos requirieron un procesamiento distinto, debido a las siguientes características: no se tenían registros de todos los años requeridos (cinco años), no se tenía información sobre el resultado final de las situaciones atendidas por Ilamadas telefónicas —-solo se tenía la clasificación inicial que utilizan los operadores telefónicos del servicio de emergencias - y la unidad de medida de los datos era distinta, porque no se sabía la intensidad de las acciones en una escala estándar, sino su cantidad reportada en periodos de años o semanas, lo cual permitió hacer comparaciones, pero con ciertas salvedades.
Los datos se dividieron entre los años que tenían registro y dicha cantidad fue dividida en 52 semanas, a fin de obtener un valor comparativo entre los dos territorios de interés. Así, se obtuvo el promedio de actividades realizadas por semana en cada colonia.

Solo se tomaron algunas de las actividades reportadas, mientras que estas fueran representativas de los conceptos esbozados páginas atrás, para evitar confusiones en las unidades policiales que las realizaban. Por ejemplo, se obtuvieron datos de los accidentes de tránsito atendidos y reportados por la ciudadanía mediante el sistema de emergencias telefónico, pero se sabe que estos son más atendidos por policías de la Unidad 911 que por las patrullas territoriales, razón por la cual fueron obviados para la investigación.

A manera de resumen, en la tabla 2 se señala el detalle puntual de los aspectos metodológicos clave.

Tabla 2

Aspectos metodológicos clave

Aspecto metodológico Investigación uno (ANSP, 2019)

Investigación dos (PNC, 2020)

Investigación mixta de carácter no experimental con recolección de datos trasversal. Datos del primer semestre de 2019.

Diseño

semestre de 2019.

- Geográfico: tres delegaciones policiales de ciudades importantes.

- Cognitivo: investigación explicativa.

Alcance

- 470 policías encuestados.

- 21 policías entrevistados.

- 67 líderes comunitarios entrevistados.

Instrumentos de recolección de datos

- Encuesta.

- Guías de entrevistas.
Investigación mixta de carácter no experimental con recolección de datos trasversal. Datos de los años 2015-2019.

- Geográfico: dos colonias urbanas de la ciudad capital, San Salvador.

- Cognitivo: investigación explicativa.

- Actividades reportadas por dos puestos policiales con patrullas territoriales fijas: 39 policías en ambos puestos.

- 20 policías entrevistados.

- 6 líderes comunitarios entrevistados.

- Solicitud presentada a la oficina de acceso a la información pública.

- Guías de entrevistas.-

El autor participó en ambas investigaciones. Se tomaron los datos de esas investigaciones y se procesaron de

Procedimiento para acuerdo con la matriz de análisis expuesta en la sección anterior, a fin de evidenciar la utilidad, usos, límites y este artículo alcances de la clasificación de actividades propuesta. Se trata de un artículo de naturaleza metodológica con fines explicativos.

Los resultados de los estudios mencionados pueden encontrarse en la siguiente sección, así como una breve lectura de la información que arrojan los datos.

\section{Resultados}

Como se mencionó antes, en la primera investigación (ANSP, 2019) se realizó una recolección de datos pri- marios de acuerdo con un diseño de actividades externo al que maneja la PNC, con el objetivo de establecer la intensidad de algunas actividades policiales y evaluarlas en una escala estándar, fijando una referencia temporal arbitraria y circunstancial. La semana previa a la recolección de datos, así, se formuló la siguiente pregunta a manera de encuesta a los miembros de la institución: ¿Con qué frecuencia realizó las siguientes actividades, durante la semana pasada? (ANSP, 2019). 
Se consideraron tres actividades por cada categoría de interés:

1. Actividades preventivas-reactivas: (i) recibir denuncias de la ciudadanía, (ii) ayudar en emergencias a la ciudadanía y (iii) atender problemas de convivencia ciudadana.

2. Actividades preventivas-proactivas: (i) participar en reuniones comunitarias, (ii) impartir charlas en centros escolares y (iii) realizar visitas casa por casa.

3. Actividades represivas-proactivas: (i) registrar personas, (ii) fichar personas y (iii) capturar personas.

Vale la pena aclarar que esta última opción de capturar personas incluía capturas flagrantes y por orden judicial. Sin embargo, luego de una reflexión teórica, posterior a la realización del estudio, se consideró que la captura, para que sea proactiva, debe derivarse de registros proactivos. Si la captura es flagrante y no se especifica, no se sabe si emerge del patrullaje no dirigido, por lo cual se considera reactiva. Asimismo, la captura derivada de una orden judicial tiene más probabilidades de ser proactiva.

Los resultados fueron convertidos en valores numéricos en una escala del 1 a 5 , donde 1 significaba nunca y 5 siempre. Cada actividad obtuvo un puntaje en esa escala y los puntajes fueron promediados entre las actividades de las mismas categorías; luego, los puntajes generales por categorías fueron comparados para realizar el análisis.

Figura 1

Frecuencia de las actividades policiales en una escala del 1 al 5

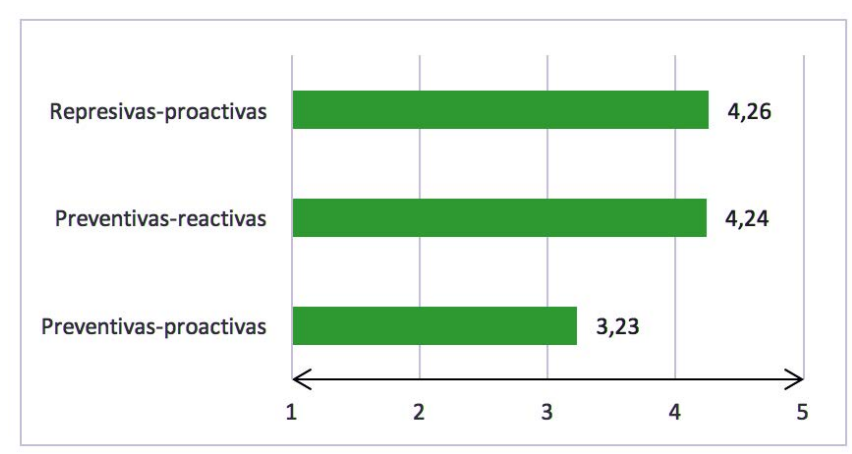

Nota. Tomado de ANSP (2019).

La desviación estándar para la primera categoría del gráfico fue de 0.85 , para la segunda 0.76 y para la tercera 1.0 .

Adicionalmente, las actividades que obtuvieron más intensidad de frecuencia, en la categoría siempre, fueron: (i) ayudar a la ciudadanía en emergencias (82\%), (ii) registrar personas $(81 \%)$, (iii) atender problemas de convivencia ciudadana (61\%) y (iv) fichar personas ( $48 \%$ ).

Por otra parte, las actividades con menos intensidad, en la categoría nunca, fueron: (i) charlas en centros escolares ( $21 \%)$, (ii) asistir a reuniones con organizaciones comunitarias $(11 \%)$ y (iii) recibir denuncias $(11 \%)$.

Los datos obtenidos permiten observar la intensidad de las actividades por medio de la escala, así como la dispersión de los datos respecto de la media. En relación a ello se pueden realizar algunas afirmaciones:

a. El personal policial se relaciona con la ciudadanía con mayor intensidad en actividades donde hay predisposición al uso de la fuerza y sobre asuntos con implicación penal.

b. La dualidad de la función policial es patente. Esto es prácticamente connatural a los cuerpos de policía y se expresa por medio de las prácticas preventivasreactivas, fundamentalmente relacionadas con servicios, las cuales, empero, no permiten desplegar una estrategia de acercamiento a la población bajo un concepto preventivo más amplio. Nótese que la dispersión de este grupo de actividades es menor, lo cual representa un campo de acción bastante trasversal para todos los policías.

c. Las actividades preventivas-proactivas son realizadas solamente por una fracción pequeña del personal policial, y no se han llegado a extender de manera general, tal como lo pretendía la PNC, argumentando la implementación de una filosofía de policía comunitaria que debía ser aplicada por todos los elementos (PNC, 2010). Es el grupo de actividades que tiene más dispersión respecto a la media.

Sin embargo, la escala que se utilizó no consideró la duración de las actividades y se concentró en la percepción de la frecuencia con que estas se realizaban, inhabilitando analizar por qué las cantidades de algunas actividades no son tan abundantes comparadas con otras.

En cuanto al segundo estudio, habida cuenta de las diferencias en la recolección de datos, fundamentalmente porque se trata de una fuente institucional (secundaria) mediada por criterios de registro y clasificación externos al marco de análisis utilizado, se agruparon las actividades por afinidades en las categorías prefijadas, según la naturaleza de las mismas. Además, tuvo que establecerse un promedio que permitiera hacer comparaciones. 
Los datos obtenidos pueden observarse en las tablas que se presentan a continuación.

Tabla 3

Actividades policiales de interés realizadas en la colonia A (caso de control territorial de una pandilla)

\begin{tabular}{|c|c|c|c|c|c|c|c|}
\hline $\begin{array}{l}\text { Actividades } \\
\text { atendidas/ realizadas }\end{array}$ & 2015 & 2016 & 2017 & 2018 & 2019 & Total & $\begin{array}{l}\text { Promedio } \\
\text { semanal }\end{array}$ \\
\hline \multicolumn{8}{|c|}{ Actividades represivas-reactivas } \\
\hline Operativos & 90 & 49 & 45 & 35 & 46 & 265 & 1.0 \\
\hline Agresiones verbales y físicas & 7 & 9 & 8 & 4 & 13 & 41 & 0.2 \\
\hline Maras/actos vandálicos & 37 & 24 & 8 & 10 & 6 & 85 & 0.3 \\
\hline \multirow[t]{2}{*}{ Detenciones } & 5 & 2 & 30 & 20 & 37 & 94 & 0.4 \\
\hline & & & & & & Global & 0.5 \\
\hline \multicolumn{8}{|c|}{ Actividades represivas-proactivas } \\
\hline Registro de personas & & & & 4326 & 5242 & 9568 & 92.0 \\
\hline Registro de vehículos & & & & 2215 & 2436 & 4651 & 44.7 \\
\hline $\begin{array}{l}\text { Levantamiento de fichas de contacto } \\
\text { ciudadano (fichaje) }\end{array}$ & & & & 46 & 52 & 98 & 0.9 \\
\hline $\begin{array}{l}\text { Operativos de Intervención Rápida } \\
\text { (OIR) }\end{array}$ & & & 15 & 38 & 45 & 98 & 0.5 \\
\hline \multirow[t]{2}{*}{ Operativos de Cierre (OC) } & & & & 12 & 25 & 37 & 0.2 \\
\hline & & & & & & Global & 27.7 \\
\hline \multicolumn{8}{|c|}{ Actividades preventivas-reactivas } \\
\hline Emergencias médicas & 22 & 27 & 27 & 24 & 14 & 114 & 0.4 \\
\hline Alteraciones al orden público & 16 & 19 & 25 & 15 & 22 & 97 & 0.4 \\
\hline \multirow[t]{2}{*}{ Servicios a la comunidad } & 5 & 13 & 12 & 12 & 13 & 55 & 0.2 \\
\hline & & & & & & Global & 0.3 \\
\hline \multicolumn{8}{|c|}{ Actividades preventivas-proactivas } \\
\hline Charlas en centros escolares & & & 75 & 84 & 87 & 246 & 1.6 \\
\hline \multirow[t]{2}{*}{$\begin{array}{c}\text { Jornadas de Casa Segura (visita } \\
\text { domiciliar) }\end{array}$} & & & 78 & 68 & 83 & 229 & 1.5 \\
\hline & & & & & & Global & 1.5 \\
\hline
\end{tabular}

Nota. Datos de PNC (2020).

Tabla 4

Actividades policiales de interés realizadas en la colonia B (caso de buenas prácticas de policía comunitaria)

\begin{tabular}{ccccccccc}
$\begin{array}{c}\text { Actividades } \\
\text { atendidas/ realizadas }\end{array}$ & $\mathbf{2 0 1 5}$ & $\mathbf{2 0 1 6}$ & $\mathbf{2 0 1 7}$ & $\mathbf{2 0 1 8}$ & $\mathbf{2 0 1 9}$ & Total $\begin{array}{c}\text { Promedio } \\
\text { semanal }\end{array}$ \\
\hline Actividades represivas-reactivas & & & & \\
\hline Operativos & 76 & 68 & 12 & 17 & 25 & $\mathbf{1 9 8}$ & 0.8 \\
Detenciones & 7 & 12 & 30 & 49 & 45 & $\mathbf{1 4 3}$ & 0.6 \\
\hline Maras/ actos vandálicos & 27 & 23 & 20 & 6 & 5 & $\mathbf{8 1}$ & 0.3 \\
\hline
\end{tabular}




\begin{tabular}{|c|c|c|c|c|c|c|c|}
\hline $\begin{array}{c}\text { Actividades } \\
\text { atendidas/ realizadas }\end{array}$ & 2015 & 2016 & 2017 & 2018 & 2019 & Total & $\begin{array}{c}\text { Promedio } \\
\text { semanal }\end{array}$ \\
\hline \multirow[t]{3}{*}{ Agresiones verbales y físicas } & 2 & 2 & 1 & 2 & 3 & 10 & 0.0 \\
\hline & & & & & & Global & 0.4 \\
\hline & \multicolumn{7}{|c|}{ Actividades represivas-proactivas } \\
\hline $\begin{array}{l}\text { Operativos de Intervención Rápida } \\
\qquad(\mathrm{OIR})\end{array}$ & & & 25 & 87 & 96 & 208 & 1.3 \\
\hline Operativos de Cierre (OC) & & & 27 & 35 & 83 & 145 & 0.9 \\
\hline Registro de personas & & & & 5525 & 6125 & 11650 & 112.0 \\
\hline Registro de vehículos & & & & 1365 & 1875 & 3240 & 31.2 \\
\hline \multirow[t]{2}{*}{$\begin{array}{l}\text { Levantamiento de fichas de contacto } \\
\text { ciudadano (fichaje) }\end{array}$} & & & & 60 & 72 & 132 & 1.3 \\
\hline & & & & & & Global & 29.3 \\
\hline \multicolumn{8}{|c|}{ Actividades preventivas-reactivas } \\
\hline Emergencias médicas & 35 & 32 & 28 & 17 & 36 & 148 & 0.6 \\
\hline Alteraciones al orden público & 32 & 8 & 10 & 12 & 64 & 126 & 0.5 \\
\hline \multirow[t]{2}{*}{ Servicios a la comunidad } & 16 & 5 & 6 & 3 & 18 & 48 & 0.2 \\
\hline & & & & & & Global & 0.4 \\
\hline \multicolumn{8}{|c|}{ Actividades preventivas-proactivas } \\
\hline Charlas en centros escolares & & & 46 & 35 & 52 & 133 & 0.9 \\
\hline \multirow[t]{2}{*}{$\begin{array}{l}\text { Jornadas de Casa Segura (visita } \\
\text { domiciliar) }\end{array}$} & & & 92 & 78 & 76 & 246 & 1.6 \\
\hline & & & & & & Global & 1.2 \\
\hline
\end{tabular}

Nota. Datos de PNC (2020).

A partir de los datos procesados, mediante la clasificación de actividades propuesta, es posible realizar las siguientes afirmaciones:

En ambos lugares las prácticas policiales demuestran un posicionamiento de la policía de manera proactiva frente a su objeto de acción, así como un acercamiento predominantemente represivo; no obstante, en cada lugar se tienen resultados de seguridad distintos, y parece que la diferencia está fuera de la información contenida en los cuadros.

a. A pesar de la implementación de un trabajo policial orientado a la comunidad, la colonia B no refleja variaciones significativas entre el promedio de las actividades preventivas-proactivas ni represivas-proactivas. Sin embargo, aquí hay un límite derivado de los datos disponibles. Por medio de la información cualitativa recolectada a través de entrevistas a policías y líderes comunitarios, sí se verificó la realización de reuniones periódicas entre el jefe local de la policía y la directiva comunitaria, solo que estas no se contabilizaron en el reporte cuantitativo entregado. En la colonia A, por otra parte, no se existe ningún nivel de coordinación entre policía y los líderes comunitarios.

b. Al profundizar en ello, se descubre una diferencia importante entre las dos colonias respecto a esa actividad en particular, que es fundamental para realizar un trabajo policial basado en la comunidad y puede conllevar diferencias cualitativas respecto a cómo se realizan el resto de actividades, considerando la justicia procedimental al realizar los registros de personas, por ejemplo. Esto según lo afirmaron líderes comunitarios entrevistados por el autor.

c. En ambos lugares es evidente la dualidad de funciones inherentes en cualquier cuerpo policial, consistente en gestionar la dimensión de control con la del mantenimiento de la paz (Wilson, 1968). Los énfasis de operatividad son iguales, salvo la variación cualitativa mencionada. La diferencia radica más bien en cómo 
se desarrollan las actividades y no en si se descarta alguna dimensión en particular.

\section{Discusión}

Al tratarse de un artículo que hace una propuesta metodológica, los hallazgos de ambas investigaciones demuestran la utilidad de la clasificación de actividades propuesta, tanto con los límites como con los alcances a tener en cuenta. No obstante, en términos generales, la clasificación logra los tres objetivos declarados: describir cómo la policía se relaciona con la población, cómo se posiciona frente a su objeto de acción y cómo se implementan programas policiales específicos en cuanto a las actividades que se ejecutan.

En el primer caso, se tuvo más control de los datos, pero se incurrió en una imprecisión en la clasificación de las detenciones como una actividad proactiva, sin que se tomaran en cuenta sus detalles.

En ese estudio se midió la intensidad de las prácticas de manera agregada por el reporte de policías como individuos. La recolección permitió agrupar y promediar la intensidad individual reportada, lo cual ofreció la ventaja de evaluar la dispersión de los datos. Con ello fue posible observar actividades que se realizan de manera más homogénea en su intensidad, un aspecto que aporta calidad al análisis organizativo.

En el segundo caso no se registraron datos individuales, lo cual no permitió analizar la dispersión de los mismos entre las personas ejecutantes. Sin embargo, sí se obtuvo un panorama acerca de las actividades de interés que, procesadas de la manera adecuada, permiten hacer comparaciones entre ellas.

Lo recomendable es realizar la recolección de datos directamente con policías operativos, seleccionando de manera adecuada los grupos de policías que son de interés. La inclusión de varios grupos puede dificultar el análisis, por la dispersión de datos, pues hay unidades estrictamente dedicadas a tareas específicas que registran más frecuencias en algunos aspectos que en otros.

Las unidades de patrullas asignadas a los territorios constituyen un área de trabajo que representa de mejor manera el despliegue territorial de la policía y la combinación de funciones de control, prevención y servicios, así como la cercanía con la ciudadanía.
Si se opta por trabajar con los registros oficiales de actividades, deberían ser discriminados aquellos datos que ofrecen ambigüedad, para ser incluidos en las categorías prefijadas, porque estos podrían contaminar el análisis global. Si su clasificación no es segura, es preferible descartarlas.

Se recomienda iniciar con un análisis de datos exploratorio, a fin de evaluar las actividades policiales que más se realizan, cualesquiera que sean, para discutir su inclusión en alguna categoría y evitar que se descarten a priori fracciones importantes del trabajo policial.

En cualquiera de los escenarios, ya sea que se puedan realizar encuestas a policías o solo se disponga de datos oficiales, se recomienda complementar el análisis con datos de la ciudadanía, perspectiva que es imprescindible en cualquier ejercicio de evaluación policial (Tudela, 2012).

Si se cuenta con más investigaciones (futuros trabajos) que utilicen la clasificación propuesta, podría evaluarse si la frecuencia de algunas actividades se mantiene en rangos más o menos estables. Es decir que, a manera de hipótesis, siempre existirá una cuota importante de actividades reactivas que son inherentes y connaturales, mientras que seguramente las acciones preventivas-proactivas se mantendrán rezagadas de las represivas-proactivas, porque las primeras no son desarrolladas por la generalidad de los policías y su realización toma más tiempo, de modo que su registro siempre será inferior, comparado con otras más cortas, bien sea que se tome como unidad de medida la intensidad estimada por los policías o los datos oficiales.

Sin embargo, no se puede afirmar que todos los cuerpos de policía sean igual de proactivos o igual de represivos, algo que solo podría observarse al analizar más datos y compararlos.

Además, es importante aproximarse a las variables que modifican de manera significativa la realización de unas actividades más que otras, como por ejemplo: el contexto territorial y la incidencia delictiva, las prioridades políticas de la policía para combatir el crimen, la confianza de la ciudadanía para denunciar, los recursos humanos y materiales de las patrullas territoriales, entre otras. Para esto, deben analizarse datos cuantitativos y cualitativos adicionales de manera complementaria.

Para darle contexto a las actividades de tipo reactivo, debe analizarse de manera especial el entono territorial 
en su sentido amplio, mientras que, para las actividades proactivas, debe ponerse atención en las fuentes internas de acción, tanto de tipo organizativo como de iniciativa de los policías operativos.

Finalmente, se esbozarán las siguientes lecturas genéricas frente a escenarios donde predominen ciertos grupos de actividades:

Si predominan las actividades represivas, esto indica una interacción tensa con la ciudadanía donde la mayoría de los encuentros ocurren mediados por la violencia; resulta clave evaluar si las intervenciones policiales están orientadas por criterios de justicia procedimental, porque de lo contrario, se estaría gestando descontento ciudadano al fomentar procesos de victimización por parte de la policía hacia la población, lo cual causa desconfianza y falta de legitimidad de la institución.

Si las acciones represivas predominantes son de tipo proactivo, entonces ello indica direccionamientos organizativos hacia el sostenimiento y la intensidad de tales acciones, con el propósito (subjetivo) de exhibir operatividad y disuadir objetivamente a la criminalidad. Estas actividades pueden llegar a desviarse de sus objetivos si se concentran contra ciertos grupos poblacionales (Reiner, 2012).

En caso de que predominen acciones de tipo preventivo, esto implica un acercamiento menos violento con la ciudadanía, en un contexto de menos incidencia delictiva o de un direccionamiento organizativo para procurarlo; sería poco usual que la policía se concentre en ello en contextos con alta criminalidad (Monjardet, 2010).

Generalmente, por su naturaleza auxiliar y disponibilidad permanente, las actividades preventivas-reactivas siempre se realizarán en una proporción alta, salvo que la ciudadanía no confíe en los servicios o existan otras instancias territoriales más eficaces a quienes recurrir en emergencias.

Las actividades preventivas-reactivas pueden ser un paliativo frente a la intensidad de las actividades represivas, a fin de hacer más evidente la dualidad policial y matizar la imagen opresiva que puedan generar estas últimas; con todo, por sí solas, no son capaces de generar confianza más allá de lo circunstancial.

La prevención proactiva sí permite desplegar una estrategia de prevención de mediano y largo plazo. Esta, como se ha visto, puede combinarse incluso con actividades de tipo represivas-proactivas, debido a que no son los mismos policías quienes las realizan generalmente. Sin embargo, si no se cuidan aspectos de justicia procedimental, seguramente la prevención proactiva sería infructuosa o saboteada por la misma policía.

La predominancia de este último grupo de actividades indicaría que la policía habría logrado motivar a buena parte de su personal, así como disuadir y contener los delitos en un nivel adecuado para que la atención represiva no eclipse el desarrollo proactivo de acciones preventivas que requieren más tiempo y dedicación.

Todos los escenarios pueden conducir a tomar decisiones importantes desde las jefaturas policiales, tales como: fortalecer los mecanismos de control interno para procurar justicia procedimental, gestionar el talento humano para la designación de tareas según sus competencias, volver más integral la asignación de tareas diarias a fin de procurar complementariedad de áreas proactivas, fortalecer y orientar la supervisión operativa de patrullas, entre otros aspectos.

\section{Conclusiones}

Como conclusión, debe tenerse en cuenta que la línea general de análisis, más allá de que predomine un tipo de actividad sobre otra, es la dualidad de las funciones policiales y el análisis sobre cómo se articulan, complementan o separan. Tanto la policía como la ciudadanía requieren un equilibrio de ambas dimensiones: la ciudadanía necesita que sean atendidas sus demandas emergentes, los problemas de seguridad asentados en los territorios y que se garanticen sus derechos; la policía necesita que no se desvíe su complementariedad, su identidad y la integralidad de sus misiones.

Además, analizar la dualidad de la dimensión reactiva y proactiva es igual de importante, tanto para la ciudadanía como para la policía. En general, las actividades proactivas deberían tener frecuencias importantes en la operatividad de la organización, indicación de que la policía no se posiciona solo como observador; sin embargo, estas no conducen por sí solas a mejores resultados. El análisis de esta dimensión recuerda que la acción policial debe tener iniciativa, pero que tampoco opera sobre realidades inertes: aquí corresponde evaluar el diseño, flexibilidad y asertividad de la proactividad, más que la definición de una estrategia inamovible. 


\section{Agradecimientos}

La revisión bibliográfica necesaria para este artículo fue posible gracias a una beca otorgada por AUIP (Asociación Universitaria Iberoamericana de Postgrado) para realizar la tesis doctoral del autor en la Universidad de Málaga (España).

\section{Referencias}

Academia Nacional de Seguridad Pública [ANSP]. (2019). Percepción ciudadana sobre el trabajo de prevención de la Policía Nacional Civil de El Salvador. Academia Nacional de Seguridad Pública.

Aparicio, J. (2017). El proceso de unificación, centralización y amalgamación de los cuerpos de policía en América. [Tesis doctoral, Universidad Externado de Colombia].

Bayley, D. (2010). Modelos de actividad policial. Un análisis comparativo. Prometeo Libros.

Cano, I. (2003). La policía y su evaluación. Propuestas para la construcción de indicadores de evaluación en el trabajo policial. Centro de Estudios para el DesarroIlo, Área Seguridad Ciudadana. http://www.observatoriodeseguranca.org/files/ignacio\%20cano.pdf

Carrington, P. J.; \& Schulenberg, J. L. (2003). Police discretion with young offenders. Department of Justice Canada. https://www.unicef.org/tdad/canadapolicediscretionyouthoffenders03(1).pdf

Dadds, V.; \& Scheide, T. (2000). Police performance and activity measurement. ACT: Australian Institute of Criminology. http://citeseerx.ist.psu.edu/viewdoc/downl oad?doi=10.1.1.552.464\&rep=rep1 \&type=pdf

Famega, C. (2003). Discretion or direction? An analysis of patrol officer downtime [PhD. Dissertation, University of Cincinnati] https://etd.ohiolink.edu/apexprod/rws_ olink/r/1501/10?p10_etd_subid=79342\&clear $=10$

Famega, C. N. (2005). Variation in officer downtime: A review of the research. Policing:Int'IJ. Police Strat. \& Mgmt., 28, 388. https://doi.org/10.1108/13639510510614528

Frank, J., Brandl, S. G.; \& Cory Watkins, R. (1997). The content of community policing: A comparison of the daily activities of community and "beat" officers. Poli- cing: An International Journal, 20(4), 716-728. https:// doi.org/10.1108/13639519710368116

Leggett, T. (2003). What do the police do? Performance measurement and the SAPS. Institute for Security Studies Papers, 66. https://hdl.handle.net/10520/EJC48954

Manning, P. K. (2011). Contingencias policiales. Prometeo Libros.

Medina, J. (2013). Políticas y estrategias de prevención del delito y seguridad ciudadana. Edisofer, Editorial BdeF.

Mohor, A. (2007). Uso de indicadores para evaluar el funcionamiento policial: notas y experiencias para la reforma policial en México [Documento de trabajo n. $\left.{ }^{\circ} 2\right]$. Centro de Estudios en Seguridad Ciudadana. https://www.cesc.uchile.cl/publicaciones/op_07_documentodos.pdf

Monjardet, D. (2010). Lo que hace la policía. Sociología de la fuerza pública. Prometeo Libros.

Policía Nacional Civil [PNC]. (2010). Manual de Formación de Policía Comunitaria de El Salvador. PNC.

Policía Nacional Civil [PNC]. (2020). Resolución de Información PNC-UAIP-173-2020. Ministerio de Justicia y Seguridad Pública, El Salvador.

Reiner, R. (2012). La política de la policía. Prometeo Libros.

Sung, H. E. (2006). Police effectiveness and democracy: shape and direction of the relationship. Policing: An International Journal, 29(2), 347-367 https://doi. org/10.1108/13639510610667709

Tyler, T. R. (2004). Enhancing police legitimacy. The annals of the American Academy of Political and Social Science, 593(1), 84-99. https://doi.org/10.1177 /0002716203262627

Travis, J. (1997). Measuring what matters: Part two: Developing measures of what the police do. US Department of Justice, Office of Justice Programs, National Institute of Justice. https://www.ncjrs.gov/pdffiles/167255.pdf

Tudela, P. (2012). La evaluación del desempeño de la policía explorando relaciones entre opinión pública 
y labor policial en Chile. Revista Criminalidad, 54(1), 379-404.

Webb, V. J.; \& Katz, C. M. (1997). Citizen ratings of the importance of community policing activities. Policing: An International Journal, 20(1), 7-23. https://doi. org/10.1108/13639519710161980
Wilson, J. (1968). Dilemmas of Police Administration. Public Administration Review, 28(5), 407-417. https:// doi.org/10.2307/973756 